\title{
Application of the Split Hopkinson Resonant Bar test for seismic property characterization of hydrate-bearing sand undergoing water saturation
}

\author{
Seiji Nakagawa and Timothy J. Kneafsey \\ Lawrence Berkeley National Laboratory, Berkeley, California, USA
}

\begin{abstract}
Conventional resonant bar tests allow the measurement of seismic properties of rocks and sediments at low frequencies (several kilohertz). However, the tests require a long, slender sample which is often difficult to obtain from the deep subsurface and weak and fractured formations. We present an alternative low-frequency measurement technique to the conventional resonant bar tests. This technique involves a jacketed core sample placed between a pair of long, metal extension rods with attached seismic source and receiver-the same geometry as the split Hopkinson pressure bar test for large-strain, dynamic impact experiments. Because of the added length and mass to the sample, the resonance frequency of the entire system can be lowered significantly, compared to the sample alone. The proposed "Split Hopkinson Resonant Bar (SHRB)" test is applied in two steps. In the first step, extension and torsion-mode resonance frequencies and attenuation of the system are measured. Then, numerical inversions for the compressional and shear wave velocities and attenuation are performed. We initially applied the SHRB test to synthetic materials (plastics) for testing its accuracy, then used it for measuring the seismic velocities and attenuation of a rock core containing supercritical $\mathrm{CO}_{2}$, and a sediment core while methane hydrate formed in the pore space.
\end{abstract}

\section{INTRODUCTION}

Seismic properties of fluid-filled, porous geological materials can be frequency-dependent, thus laboratory measurements should be performed at field-employed frequencies. Unfortunately, laboratory measurement of lowfrequency $(<10 \mathrm{kHz})$ seismic properties is a difficult task with traditional ultrasonic equipment and limited core size. Quasi-static techniques (e.g., [1]) allow measurements of low-frequency seismic properties continuously up to several hundreds of hertz, but such measurements are difficult and not commonly performed. Conventional resonant bar tests allow measuring seismic properties of rocks and sediments at relatively low frequencies (typically hundreds of hertz to several kilohertz) relatively easily. One disadvantage of this technique, however, is that the test requires a long, slender sample, which is often difficult to obtain from deep rock/sediment and weak and fractured formations. If the conventional resonant bar test is applied to 5- $10 \mathrm{~cm}$-long cores typically available from such formations, the resonance frequency can be as high as tens of kilohertz.

Alternatively, a composite bar, consisting of a metal extension rod (including a seismic source), jacketed rock or sediment core sample, and another metal rod (including a seismic receiver), can be used to reduce the resonance frequency of the system. The reduction in the resonance frequency increases for longer length and larger mass of the extension rods. (Tittmann [2] proposed the use of additional mass in resonant bar tests to reduce resonance frequencies.) Because this sample geometry is the same as the split Hopkinson pressure bar test used primarily for large-strain, dynamic impact experiments, we call this technique the "Split Hopkinson Resonant Bar (SHRB)" test.

In the following, we will first describe the testing methodology of the SHRB test, then our experimental setup and the measurement procedures. The principles of numerical inversion for elastic moduli and seismic velocities and attenuation from the laboratory measurements will also be discussed, along with necessary corrections to the model for removing measurement artifacts. Following the description of the method, we will present applications of the SHRB test in the laboratory. First, we will show the accuracy of the technique when applied to synthetic materials (plastics) having known material properties. Then we will apply the technique to a partially water saturated sediment core injected with methane gas to form methane hydrate within the pore space, and a subsequent water flood of the sample. Finally, we will summarize the strengths and weakness of the SHRB test and make recommendations for its use and further development.

\section{METHOD}

\subsection{General Methodology}

The SHRB test is applied in two steps. First, laboratory measurements are conducted for resonance frequencies and attenuation of the entire system (extension bars + sample). In the next step, these measurements are used as an input to a numerical inversion code for determining the Young's modulus $(E)$ and shear modulus $(G)$ of the sample itself. These moduli are combined with separately known or measured material density of the sample to determine seismic velocities and attenuation. 


\subsection{Experimental Setup}

The stainless steel extension rods of the SHRB setup have a diameter of $3.75 \mathrm{~cm}$ (1.5 inches) and a length of $40.6 \mathrm{~cm}$ (16 inches). At the end of the extension rods, a source unit (houses both compression and torsion piezoceramic sources) or a receiver unit (houses multi-component accelerometers) is attached for seismic measurements (Fig.1).

For measuring seismic properties, a rock or sediment core is placed between the extension rods. The diameter of the core is the same as the rods. The core is jacketed by a thin PVC heat-shrink tube having a thickness of $\sim 250 \mu \mathrm{m}$ to 500 $\mu \mathrm{m}$. For hard, consolidated rock samples, thin lead foil sheets are placed at the sample-rod interfaces to assure good mechanical coupling.

Through a pair of ports in the extension rods, pore fluids can be injected and extracted during the experiment, under controlled temperature and confining pressure.

Once assembled, the entire system consisting of the extension rods, source, receiver, and the jacketed core sample is suspended by steel springs within a tubular cage (Fig.1a) which is inserted in a pressure vessel. Within this vessel, the confining stress is applied via compressed nitrogen gas.

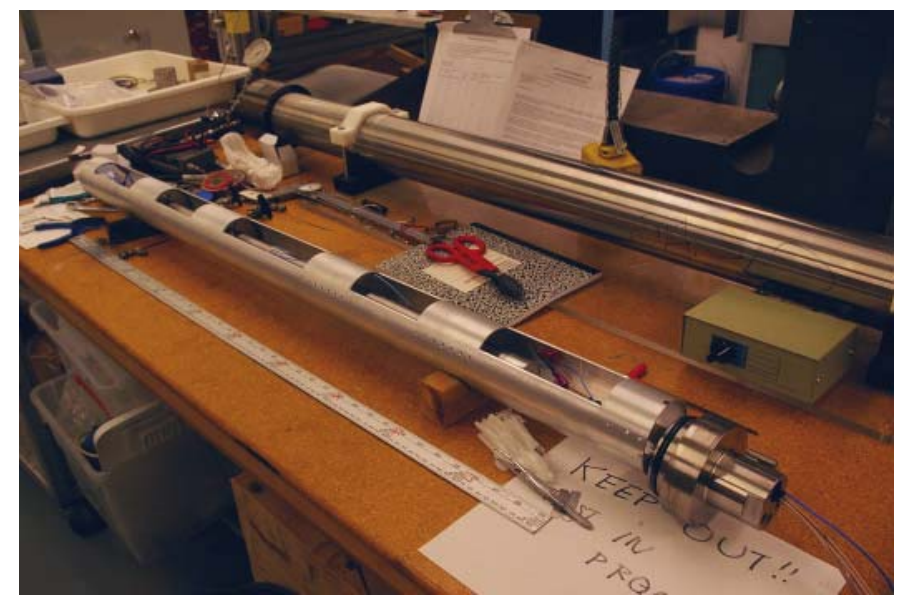

(a) Gas confining cell (top right) and a suspension cage (front) housing the sample-bar assembly

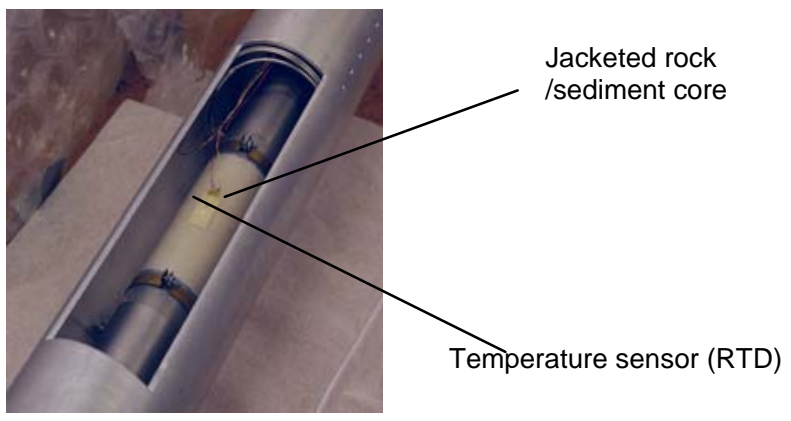

(b) Jacketed sample core placed between steel extension rods

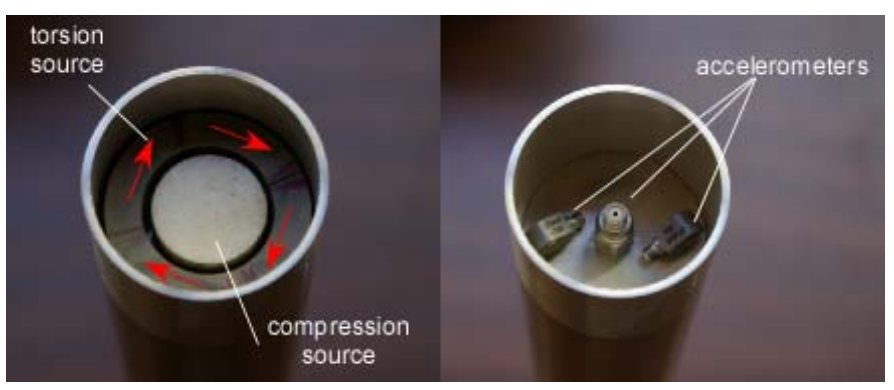

(c) Source and receiver units attached to the ends of the bars. Note that these units were later potted with high-strength epoxy for protection.

Fig. 1. SHRB test setup. 


\subsection{Laboratory Measurement}

For given temperature, confining pressure, and pore pressure conditions, both compression mode ( $E$ mode) and torsion mode ( $G$ mode) vibrations are induced in the sample. For the torsion-mode measurements, parasitic, flexural (bending)-mode vibrations which interfere with the torsion-mode resonances are reduced, by using two accelerometers canceling the flexural motions and enhancing the torsion motions at the same time.

Laboratory measurements are conducted in the same way as conventional resonant bar tests. The resonance frequencies are determined from the peak frequency of the amplitude or power spectra, and the attenuation from the half-power width of the peak (Fig.2). Currently, we measure only the fundamental modes of the compression and torsion modes, which have the lowest frequencies.

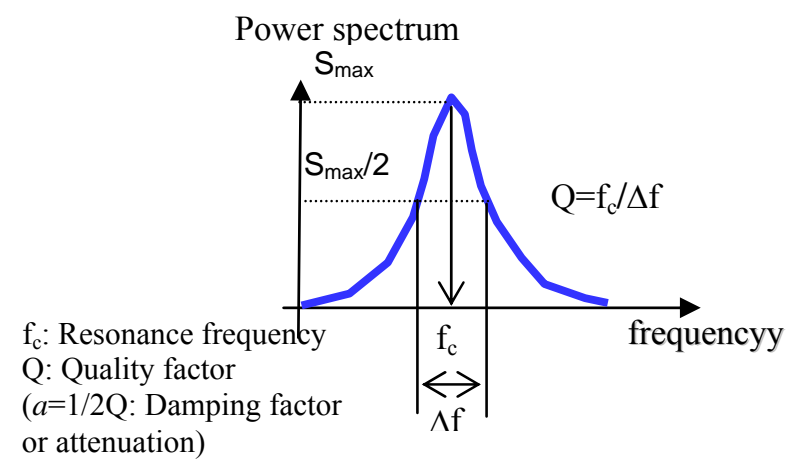

Fig. 2. Measurement of resonance frequency and attenuation from power spectra

\subsection{Numerical Inversion}

Numerical inversions are conducted using a one-dimensional acoustic wave propagation code for the each mode. The basic steps of the inversion are:

(i) Assume a complex, model elastic modulus for the sample

(ii) Apply corrections for experimental artifacts

(iii) Compute numerical resonance frequency and attenuation

(iv) Compare measured and computed resonance frequency and attenuation

(v) If the results do not match, update the model elastic modulus. Then repeat (ii)-(v).

Because this is a one-dimensional model, inversion for each mode (extension and torsion) is conducted separately.

In step (ii), the sample properties are adjusted for the jacket properties (jacket correction), and the extension rod properties are adjusted for the source and receiver mass and length. For $E$ mode, an additional correction must be applied for the effect of friction between the sample and the extension rods, which constricts the lateral expansion of the sample if the length of the sample is short compared to its diameter.

To model this effect, we first assume that the cone-shaped region within a cylindrical core where this friction effect is present, has a uniform vibration-induced lateral strain which is given by the strain of the metal extension rods at the interface. The rest of the core is at the uniaxial stress state (no vibration-induced lateral stress). Next, we further simplify the model by assuming that the axial compression of the sample can be given by a sum of the axial compression of two layers - one with the given lateral strain of the metal rods and the other under uniaxial stress state-during vibration (Fig.3). Each layer has the same volume as in the original sample. This simplification allows us to derive an expression for an effective Young's modulus of the core, as a function of the extension rod and the sample's Young's moduli, shear moduli (or Poisson's ratios), sample diameter, sample length, and the angle of the cone for the zone affected by the friction. This last parameter is a fitting parameter which can be determined using a numerical model. Through elasto-static finite-element modeling, we found that an angle of $25-27^{\circ}$ was adequate for matching the effective Young moduli from the theory and the numerical simulations, when the stiffness of the sample is much smaller than the extension rods. (The angle needs to be a function of the ratio between the elastic moduli of the sample and the rods if the sample stiffness is similar to the rods.) 


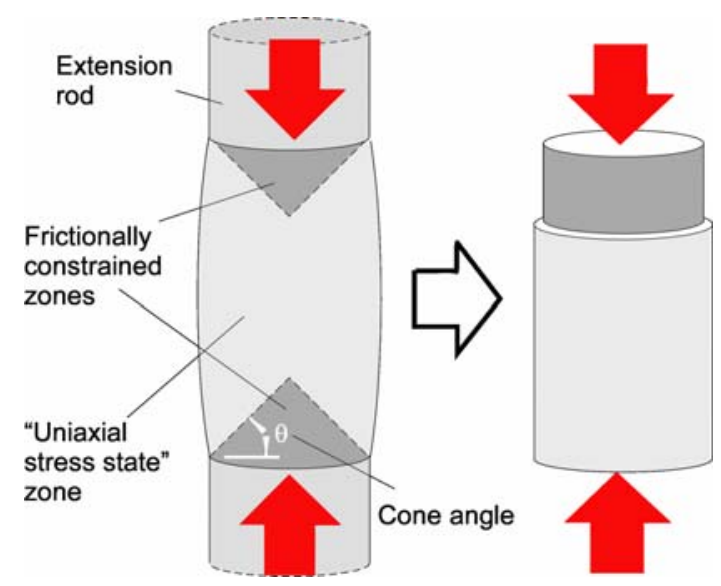

Fig. 3. Idealization of stress state within a cylindrical sample for deriving the effective Young's modulus of the sample considering the end-friction effect.

Because the end-friction correction involves adjusting the Young's modulus $E$ of the sample as a function of the shear modulus, the inversion for the complex shear modulus $G$ is conducted first, then the resulting modulus is used as a "true value" during the inversion for E. Finally, using either measured or known density of the sample, the P and S wave velocities $\left(V_{P}\right.$ and $V_{S}$, respectively) are determined from the elastic moduli. The attenuation of the waves is determined from a ratio between the real and imaginary part of the related moduli. For the P-wave modulus $H=G(4 G-E) /(3 G-E)$ and the S-wave modulus $G$, these are

$$
\begin{aligned}
& a_{P}=\frac{1}{2} \frac{\operatorname{Im}(H)}{\operatorname{Re}(H)}=\frac{1}{2 Q_{P}}, \\
& a_{S}=\frac{1}{2} \frac{\operatorname{Im}(G)}{\operatorname{Re}(G)}=\frac{1}{2 Q_{S}},
\end{aligned}
$$

where $\mathrm{Q}_{\mathrm{P}}$ and $\mathrm{Q}_{\mathrm{S}}$ are the seismic quality factors. Note that the moduli are treated as complex parameters. Also note that the resonance frequencies for the $E$ and $G$ modes are generally different, which may lead to inaccuracy in determining the material properties for a single frequency. Although this is a weakness of the method, for many geological materials, material properties are not strongly frequency dependent within a half decade of frequency band width which seems to cover both $E$ and $G$ resonance frequencies in most cases.

\section{DEMONSTRATION - ACCURACY TEST USING PLASTIC CORES}

To examine the accuracy of the SHRB test for determining the complex elastic moduli of a sample, we conducted a series of measurements using acrylic $(\mathrm{Q} \sim 25)$ and polycarbonate $(\mathrm{Q} \sim 100)$ cores with a range of lengths $(\mathrm{L}=0.95,1.9$, 3.8, 7.6, and $11.4 \mathrm{~cm})$. The determined Young's moduli $(|\mathrm{E}| \mathrm{s})$ and shear moduli $(|\mathrm{G}| \mathrm{s})$ and their attenuation $\left(a_{E}=1 / 2 \cdot \operatorname{Im}(E) / \operatorname{Re}(E)\right.$ and $a_{G}$, respectively) are shown in Fig.4, as discrete symbols connected by solid lines (To avoid the previously mentioned ambiguity in the frequency of the measurements, we do not plot velocities and their attenuation.) Because shorter core lengths result in higher resonance frequencies, the resulting moduli and attenuation are plotted as a function of measured resonance frequencies. The "true" material properties near the measurement frequencies were determined from conventional resonance tests using $\sim 1 \mathrm{~m}$ long cores of the same material. These results are shown by "X"s (for $E$ mode) and "+"s (for $G$ mode) in the plots.

Figure 4 also shows the results without applying the end-friction correction during the $E$-mode inversion (shown as discrete symbols connected by dotted lines in the plot). This demonstrates that the end-friction corrections can be very large for short cores. When Poisson's ratios are computed from these results, the difference is more dramatic (Fig.5). Without the correction, inverted Poisson's ratio of the sample can be extremely large, often exceeding 0.5 . This also implies that errors in inverted P-wave velocities can be very large if the corrections are not applied properly. 


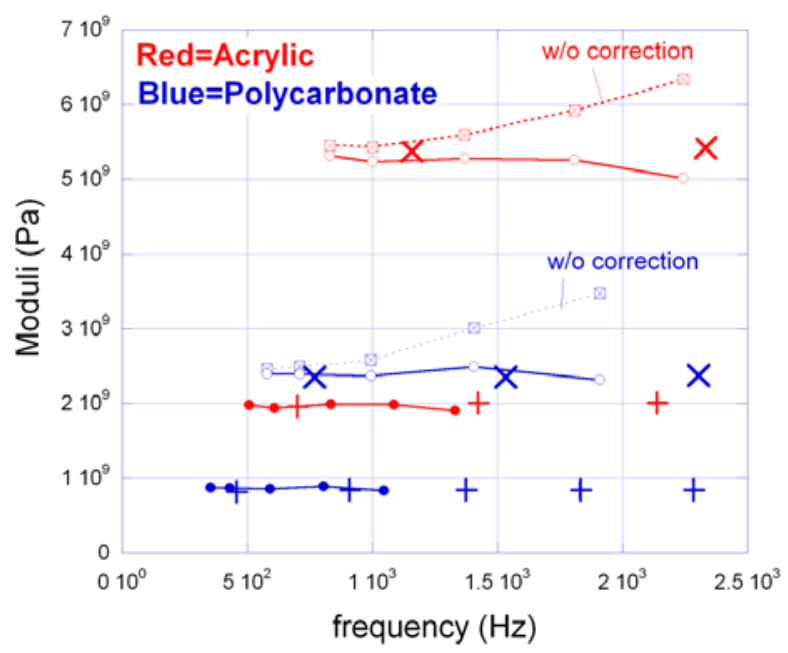

(a) Moduli $|\mathrm{E}|(\circ)$ and $|\mathrm{G}|(\bullet)$

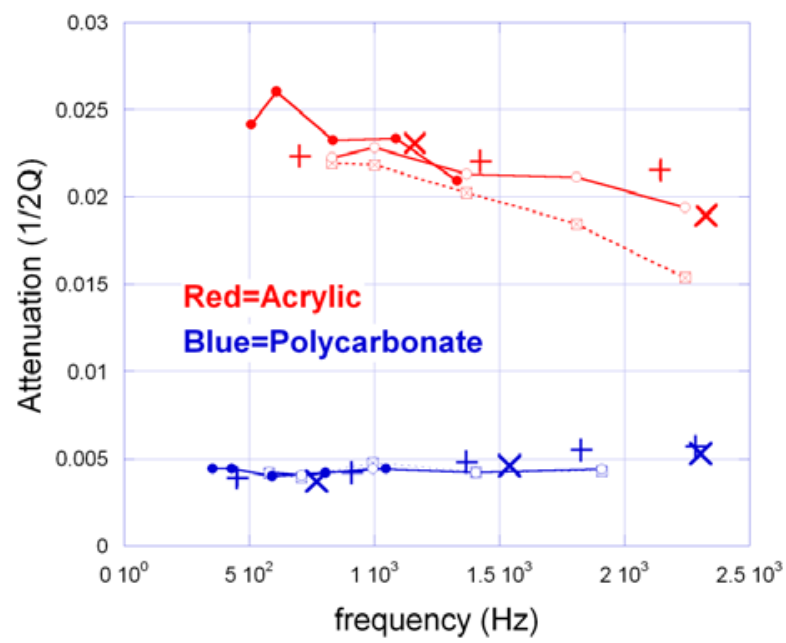

(a) E-mode attenuation (०) and G-mode attenuation $(\bullet)$

Fig. 4. Inverted moduli and related attenuation. "X" (for E-mode) and "+" (for $G$ mode) are the "true" values. The dotted lines are the results for E mode inversion without the end-friction corrections.

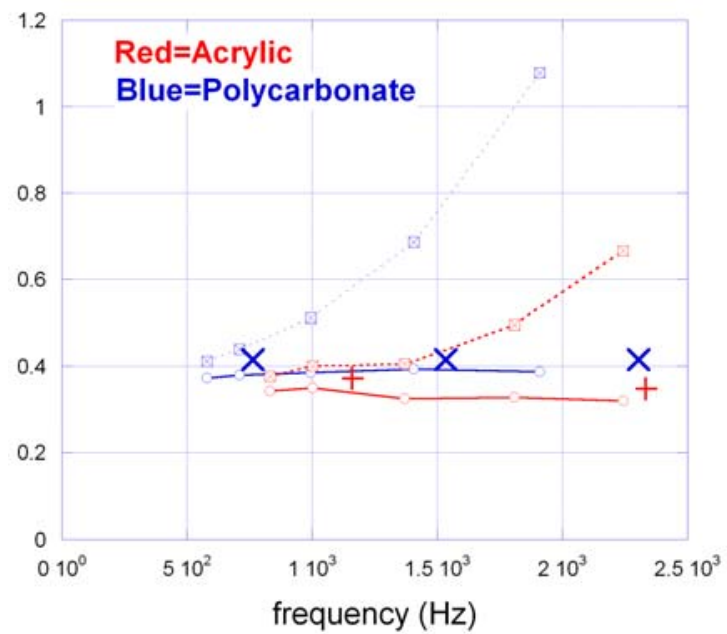

Fig. 5. Inverted Poisson's ratios. The results are plotted against the resonance frequency of the $E$ mode. 


\section{SEISMIC SIGNATURES OF METHANE HYDRATE FORMATION WITHIN PARTIALLY SATURATED SEDIMENT}

For assuring seafloor stability during off-shore oil and gas exploration and for monitoring of gas production during methane hydrate exploration, it is essential to understand the seismic signatures of methane hydrate formation and dissociation. In this example, we examined the seismic velocity and attenuation changes during the formation of methane hydrate within a partially water-saturated sand pack.

In this experiment, we used pure silica sand (US Silica, F110 sand) with a nominal grain size of $100 \mu \mathrm{m}$ and subangular grain shape. First, the sand was compacted in a PVC jacket (thickness $\sim 0.5 \mathrm{~mm}$ ) to a porosity of $35 \%$, with a small amount of distilled water $(20 \%$ of the pore volume) to a length of $7.62 \mathrm{~cm}$. Subsequently, the sample was introduced into the SHRB system, and the confining stress was applied at $6.9 \mathrm{MPa}$. At this point, the sample pore space was flushed by several pore volumes of $\mathrm{CH}_{4}$ gas and then evacuated.

At $\mathrm{t}=0$, dry methane gas was injected into the sand pack at a pressure of $5.6 \mathrm{MPa}$. The temperature of the sample, measured by a temperature sensor attached to the sample jacket, stayed within $3.5-4.5^{\circ} \mathrm{C}$ throughout the experiment. At this temperature and pressure, methane and water forms hydrate. Because the hydrate in our partially water saturated sample formed primarily within the pendular water around the grain contacts, it was expected that the hydrate formation would result in cementation of the grains, causing increases in seismic velocities and decreases in attenuation.

Figure 6a shows changes in seismic velocities inverted from measured resonances in the frequency range of $300 \mathrm{~Hz}-1.5$ $\mathrm{kHz}$. As expected, there are dramatic increases in seismic velocities attributable to hydrate formation, especially for the first 8 hours from the beginning of the experiment. The attenuation of the waves, however, exhibited unexpected behavior, as seen in Fig.6b. Instead of showing expected monotonic decreases, the attenuation initially increased rapidly to very large values $\left(a_{P} \sim 3.6 \%\right.$ and $a_{S} \sim 3.3 \%$, corresponding to $Q_{P} \sim 14$ and $\left.Q_{S} \sim 15\right)$. This large attenuation diminished quickly after reaching the peak values. After 24 hours from the start of the experiment, where the increase in velocities became very small, the level of attenuation reduced to near the initial level. (Note: We recently learned that the same changes in seismic attenuation were observed by Rees [5] via a resonant column experiment during hydrate formation.)

The observed, unusual changes in the attenuation may be explained by the morphology of hydrate formation at a grain contact. Subramanian and Sloan [6] conducted micro-scale visualization of methane-hydrate formation at a gas-water interface. This study revealed that during hydrate formation, a thin film of hydrate forms first at the interface, then microscopic, needle-shaped crystals start to grow into the water phase, until the remaining water is converted into solid. In our case, where the gas-water interface is on the pendular water at a grain contacts, this growth process results in the "liquid-to-solid transition" of the pendular water. Thus, when the hydrate film forms on the gas-water interface, the effective viscosity of the fluid becomes large, resulting in very large attenuation of seismic waves. Once the film thickens and the hydrate-water mixture at the grain contact gains rigidity, the behavior of the material becomes that of a solid, resulting in the subsequent decreases in attenuation.

This "attenuation spike" can be a very useful tool for identifying new hydrate formation within a methane gas-watersediment system. 


\section{SEISMIC SIGNATURES OF METHANE HYDRATE-BEARING SAMPLE DURING A WATER FLOOD}

Upon reaching steady state following methane hydrate formation, we injected water into the sample and continued to record the resonance properties. Adding water to a hydrate-bearing sand containing methane under stable conditions induces 1) additional hydrate formation by the addition of water and 2) hydrate dissolution at the water inlet from the fresh (methane free) water, but is also thought to change the location hydrate occupies the pore space from cementing to pore filling (Ebinuma, 2008 conference presentation). A sample containing cementing hydrate (at lower hydrate saturations) will typically be more stiff (higher resonant frequency) than the same sample with pore-filling hydrate.

Figure 7 shows the resonance frequencies over the duration of the test, from moist sand, to hydrate formation, to water injection, and finally dissociation. As discussed above, the sample becomes much stiffer upon hydrate formation (cementing). The stiffness increases somewhat over the next 100 hours or so, and the corresponding P and S-wave speeds increase as well (Figure 6). Water injection causes the resonant frequency to decline significantly, yet the resulting frequencies are still above the starting (moist sand) frequencies. This is consistent with sand with pore-filling hydrate, as the hydrate would contribute to the stiffness, yet not as much as the hydrate cementing case. Thermal dissociation caused the sample to soften approximately to the initial (moist sand) condition.

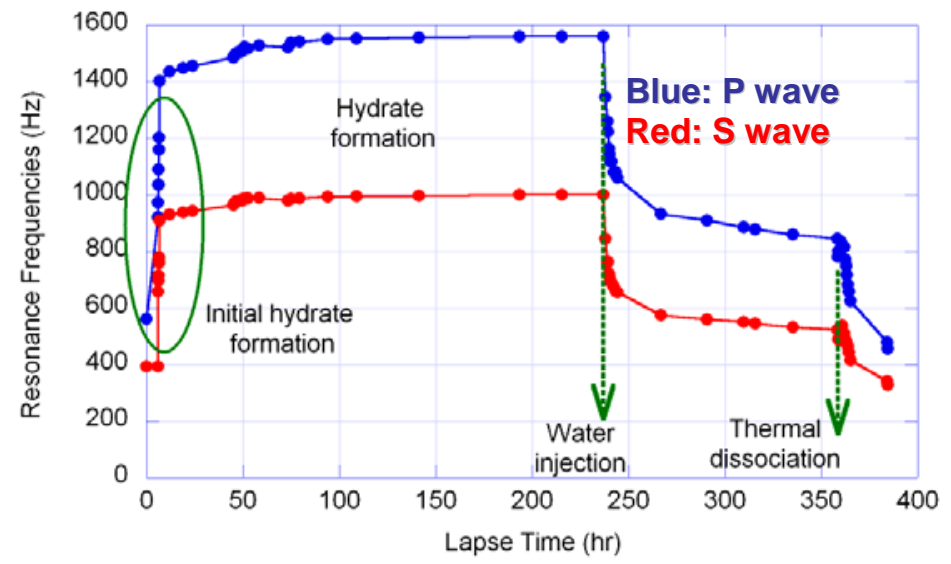

Figure 7. Evolution of measured resonance frequencies during the experiment (Test\#1).

Figure 8 presents the seismic velocities and attenuation for two water injection experiments. In Test 1, water was easily injected into the sample, whereas in Test 2, water was only slowly injected because of hydrate formation and pore space and line clogging. In both tests however, the seismic velocities decreased significantly (up to $\sim 50 \%$ of the initial velocities) in spite of the probable formation of additional hydrate in the samples from the addition of water. The attenuation rapidly increased upon water injection. These increases are probably caused by the formation of new hydrate crystals within the sample. The seemingly contradicting results - formation of additional hydrate and decreasing seismic velocities (i.e., cementation between sediment grains) - are attributed to the migration of hydrate crystals from intergranular contacts to the pore fluid. This explanation is supported by the observation by Katsuki et al $(2006,2007)$. Using glass micromodels, they showed that when water-saturated, gas hydrates (methane hydrate and $\mathrm{CO}_{2}$ hydrate) change their habit within the pore space over time. 

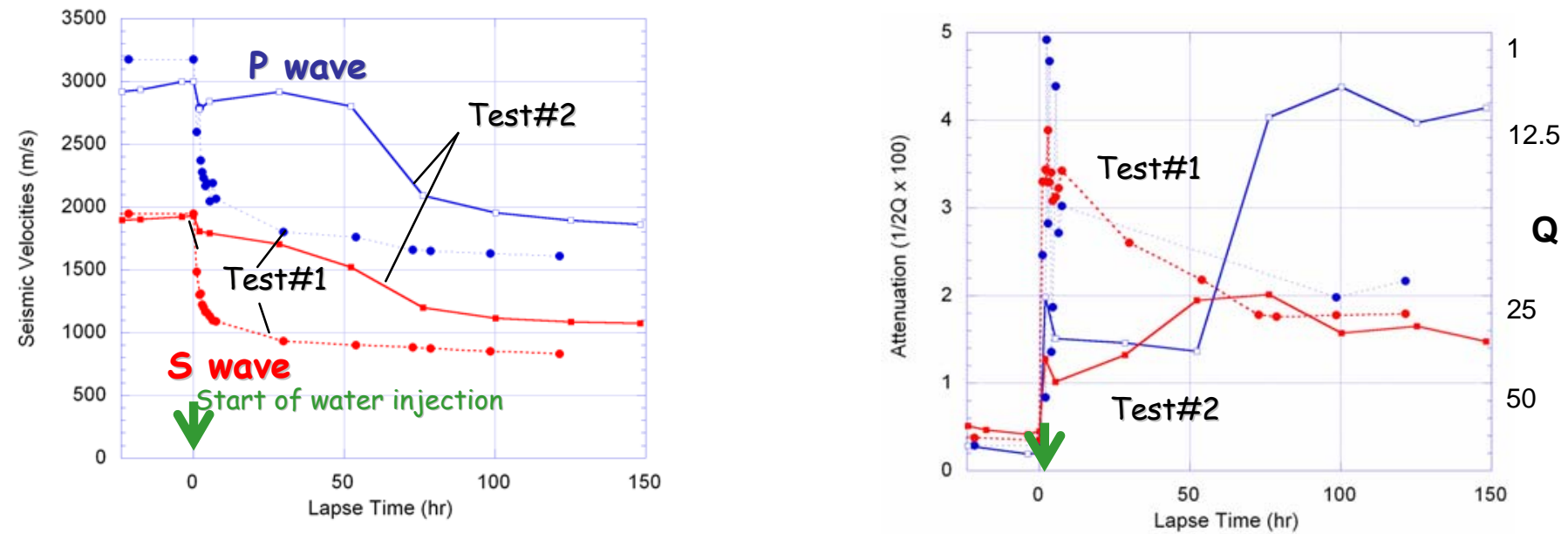

Figure 8. Seismic velocities and attenuation over the water injection period. Hydrate clogging in Test 2 resulted in slow uptake of water.

\section{CONCLUSIONS}

Split Hopkinson Resonant Bar (SHRB) tests allow us to measure seismic velocity and attenuation of small rock and sediment cores at low frequencies in the laboratory. Using the current setup, the frequency of measurement for typical rock cores is near $1 \mathrm{kHz}$. Because the SHRB test is essentially a resonant bar method, it has the same advantages as the conventional technique, including the capability to measure intrinsic (rather than scattering-induced) attenuation of the sample reliably and a high resolution for measuring small changes in the seismic properties of the sample.

In contrast, SHRB tests have some ambiguities in the frequency of measurements for computed P-wave velocity and attenuation, because these quantities are inverted from both extension and torsion-mode resonances which are measured at different frequencies. Also, because the measurement for each mode is made for only a single, lowestfrequency resonance, frequency dependency of seismic properties is not examined. This problem can be solved if multiple samples with different lengths are available, as this provides a range of resonance frequencies.

As the examples of the application indicate, the SHRB test can be a useful tool for time-lapse monitoring of seismic velocities and attenuation in the laboratory during fluid injection in rock and sediment samples. Particularly, measurements of attenuation can provide valuable information regarding the grain-scale interaction of pore fluid and mineral grains and cement.

Methane hydrate formed from water in moist sand cemented the sample together consistent with the observations of Waite et al. [7]. During hydrate formation, the attenuation of the seismic signal increased dramatically as the resonant frequency increased. We believe that this is from newly formed hydrate stiffening the sample and simultaneously causing the water in the sample to behave as a very viscous fluid. When hydrate formation was complete, the attenuation dropped significantly from the peak value. Water injection into the remaining pore space changed the resonance behavior of the sample, making it less stiff, while also temporarily increasing attenuation. We believe that the decrease in stiffness is caused by hydrate reforming away from the grain contacts. This is consistent with visual observations of hydrate behavior in transparent micromodels. The increase in attenuation is attributed to hydrate formation in the injected water. This causes the water to behave as a very viscous fluid.

Lastly, the SHRB tests can be conducted using a x-ray transparent pressure vessel, which allows real-time x-ray CT imaging of gas and fluid distribution within a sample during experiments. Currently, we are conducting such experiments to correlate the gas/fluid distribution to the seismic properties of methane-hydrate-bearing sediment and reservoir sandstone cores during an injection of supercritical $\mathrm{CO}_{2}$ to replace in-situ brines.

\section{ACKNOWLEDGMENTS}

This research has been supported by the Office of Science, Office of Basic Energy Sciences, Division of Chemical Sciences of the U.S. Department of Energy by the Assistant Secretary for Fossil Energy, Office of Natural Gas and Petroleum Technology, GEO-SEQ Program and Gas Hydrate Program, through the National Energy Technology Laboratory, under the U.S. DOE, Contract No. DE-AC02-05CH11231.

\section{REFERENCES}

1. Spencer, J.W. 1981. Stress relaxation at low frequencies in fluid saturated rocks: attenuation and modulus dispersion, $J$. Geoph. Res., 1803-1812. 
2. Tittmann, B.R. 1977. Internal friction measurements and their implications in seismic Q structure models of the crust, $A G U$ Geophys. Mong., 20, The earth's crust, 197-213.

3. E.W. Lemmon, M.O. McLinden and D.G. Friend, Thermophysical Properties of Fluid Systems, in NIST Chemistry WebBook, NIST Standard Reference Database Number 69, Eds. P.J. Linstrom and W.G. Mallard, National Institute of Standards and Technology, Gaithersburg MD, 20899, http://webbook.nist.gov

4. Biot, M.1962. Thoery of propagation of elastic waves in a fluid saturated porous solid. I. Low frequency range and II. Higher-frequency range, J. Acoust. Soc. Am., 28, 168-191.

5. Rees, E.V.L. 2009. Methane Gas Hydrate Morphology and its Effect on the Stiffness and Damping of Some Sediments, Ph.D. Thesis, U. Southampton, UK.

6. Subramanian, S., Sloan, E.D., 2002. Solubility effects on growth and dissolution of methane hydrate needles. In: Proceedings of the Fourth International Conference on Gas Hydrates, Yokohama, 856-861.

7. Waite, W. F., et al. (2004), Methane hydrate formation in partially water-saturated Ottawa sand, American Mineralogist, 89, $1202-1207$. 


\section{DISCLAIMER}

This document was prepared as an account of work sponsored by the United States Government. While this document is believed to contain correct information, neither the United States Government nor any agency thereof, nor The Regents of the University of California, nor any of their employees, makes any warranty, express or implied, or assumes any legal responsibility for the accuracy, completeness, or usefulness of any information, apparatus, product, or process disclosed, or represents that its use would not infringe privately owned rights. Reference herein to any specific commercial product, process, or service by its trade name, trademark, manufacturer, or otherwise, does not necessarily constitute or imply its endorsement, recommendation, or favoring by the United States Government or any agency thereof, or The Regents of the University of California. The views and opinions of authors expressed herein do not necessarily state or reflect those of the United States Government or any agency thereof or The Regents of the University of California.

Ernest Orlando Lawrence Berkeley National Laboratory is an equal opportunity employer. 